\title{
FINITE DIFFERENCE METHOD FOR GENERALIZED ZAKHAROV EQUATIONS
}

\author{
QIANSHUN CHANG, BOLING GUO, AND HONG JIANG
}

\begin{abstract}
A conservative difference scheme is presented for the initialboundary value problem for generalized Zakharov equations. The scheme can be implicit or semiexplicit depending on the choice of a parameter. On the basis of a priori estimates and an inequality about norms, convergence of the difference solution is proved in order $O\left(h^{2}+\tau^{2}\right)$, which is better than previous results.
\end{abstract}

\section{INTRODUCTION}

The Zakharov equations [20]

$$
\begin{gathered}
i E_{t}+E_{x x}-N E=0, \\
\frac{1}{\lambda^{2}} N_{t t}-\left(N+|E|^{2}\right)_{x x}=0
\end{gathered}
$$

describe the propagation of Langmuir waves in plasmas. Here the complex unknown function $E$ is the slowly varying envelope of the highly oscillatory electric field, and the unknown real function $N$ denotes the fluctuation of the ion density about its equilibrium value.

The global existence of a weak solution for the Zakharov equations in one dimension is proved in [19], and existence and uniqueness of a smooth solution for the equations are obtained provided smooth initial data are prescribed.

Numerical methods for the Zakharov equations are studied only in [5, 9, 10, and 15]. A spectral method is used to compute solitary waves and the collision of two solitary waves in [15]. In [9, 10], Glassey considered an energy-preserving implicit difference scheme for the equations and proved its convergence in order $O(h+\tau)$. In [5], we propose a new conservative difference scheme which involves a parameter $\theta, 0 \leq \theta \leq \frac{1}{2}$; when $\theta=\frac{1}{2}$, the new scheme is identical to Glassey's scheme. For $\theta=0$, the new scheme is semiexplicit, explicit in $N$, but implicit in $E$. Numerical experiments demonstrate that the new scheme with $\theta=0$ is more accurate and efficient compared to $\theta=\frac{1}{2}$. Convergence of these schemes is proved in order $O(h+\tau)$ in [5, 9, and 10], while the order of the truncation errors is $O\left(h^{2}+\tau^{2}\right)$.

Received by the editor September 16, 1993 and, in revised form, December 28, 1993 and April 7, 1994.

1991 Mathematics Subject Classification. Primary 65M06, 65M12.

Key words and phrases. Difference scheme, Zakharov equation, convergence. 
For suitable initial data, the solution of the initial value problem for (1.1)(1.2) converges as $\lambda \rightarrow \infty$ to a solution of the cubic nonlinear Schrödinger equation

$$
i E_{t}+E_{x x}+|E|^{2} E=0
$$

(see $[1,17])$.

The generalized nonlinear Schrödinger equation

$$
i E_{t}+E_{x x}+f\left(|E|^{2}\right) \cdot E=0
$$

has been considered in physics (see, for example, [2, 3, and 11]). Here, $f(s)=$ $s^{p}(p>0), f(s)=1-e^{-s}, f(s)=\frac{s}{1+s}$ or $f(s)=\ln (1+s)$. Existence and uniqueness of the generalized solution for the equation (1.4) have been obtained and numerical methods for (1.4) have been studied (see [4, 6, and 18]).

The generalized Zakharov equations are also considered in [21]. In the present paper, we consider the following initial-boundary value problem of generalized Zakharov equations in one dimension:

$$
\begin{gathered}
i E_{t}+E_{x x}=N f\left(|E|^{2}\right) E, \quad x_{L}<x<x_{R}, T \geq t>0, \\
N_{t t}-N_{x x}=\frac{\partial^{2}}{\partial x^{2}}\left(F\left(|E|^{2}\right)\right),
\end{gathered}
$$

where

$$
\begin{gathered}
f \in C^{\infty}\left(R^{+}\right), \quad F(s)=\int_{0}^{s} f(\tau) d \tau \\
E(x, 0)=E^{0}(x), \quad N(x, 0)=N^{0}(x), \quad N_{t}(x, 0)=N^{1}(x), \\
\left.E\right|_{x=x_{L}}=\left.E\right|_{x=x_{R}}=0,\left.\quad N\right|_{x=x_{L}}=\left.N\right|_{x=x_{R}}=0,\left.\quad u\right|_{x=x_{L}}=\left.u\right|_{x=x_{R}}=0,
\end{gathered}
$$

and the potential function $u$ is given by

$$
u_{x x}=N_{t} .
$$

Moreover, we supplement (1.5)-(1.10) by imposing the compatibility condition

$$
\int_{x_{L}}^{x_{R}} N^{1}(x) d x=0 .
$$

We propose a conservative difference scheme with parameter $\theta$ of the generalized Zakharov equations. The difference scheme conserves two conservation laws that the differential equations possess. For $\theta=0$, the scheme is semiexplicit. We will prove the convergence of the difference solution for all $\theta \in\left[0, \frac{1}{2}\right]$ in order $O\left(h^{2}+\tau^{2}\right)$, which is consistent with the order of the truncation error of the difference scheme. This improves the results of order $O(h+\tau)$ which were given in $[5,9]$.

In $\S 2$, we describe the difference scheme and its basic properties. Some a priori estimates and the proof of convergence of the difference scheme are given 
in §3. Long and highly technical proofs of two lemmas in $\S 3$ are placed in the Supplement section at the end of this issue.

\section{FINITE DIFFERENCE SCHEME}

We consider a finite difference method for the problem (1.5)-(1.11). As usual, the following notations are used:

$$
\begin{aligned}
x_{j}=x_{L}+j h, \quad t^{n}=n \cdot \tau, \quad 0 \leq j \leq J=\left[\frac{x_{R}-x_{L}}{h}\right], \quad n=0,1,2, \ldots,\left[\frac{T}{\tau}\right], \\
E(j, n) \equiv E\left(x_{j}, t^{n}\right), \quad N(j, n) \equiv N\left(x_{j}, t^{n}\right), \\
E_{j}^{n} \sim E(j, n), \quad N_{j}^{n} \sim N(j, n), \\
\left(W_{j}^{n}\right)_{x}=\frac{1}{h}\left(W_{j+1}^{n}-W_{j}^{n}\right), \quad\left(W_{j}^{n}\right)_{\bar{x}}=\frac{1}{h}\left(W_{j}^{n}-W_{j-1}^{n}\right), \\
\left(W_{j}^{n}\right)_{t}=\frac{1}{\tau}\left(W_{j}^{n+1}-W_{j}^{n}\right), \quad\left(W_{j}^{n}\right)_{\bar{t}}=\frac{1}{\tau}\left(W_{j}^{n}-W_{j}^{n-1}\right), \\
\left\|W^{n}\right\|_{2}^{2}=h \sum_{j=1}^{J}\left|W_{j}^{n}\right|^{2}, \quad\left\|W^{n}\right\|_{\infty}=\sup _{1 \leq j \leq J}\left|W_{j}^{n}\right|, \\
r=\frac{\tau}{h}, \quad \beta=\frac{\tau}{h^{2}},
\end{aligned}
$$

and in this paper $C$ denotes a general constant, which may have different values in different occurrences. Thus, our scheme is written as

$$
\begin{gathered}
i\left(E_{j}^{n+1}\right)_{\bar{t}}+\frac{1}{2}\left(\left(E_{j}^{n+1}\right)_{x \bar{x}}+\left(E_{j}^{n}\right)_{x \bar{x}}\right) \\
=\frac{1}{4}\left(N_{j}^{n+1}+N_{j}^{n}\right)\left(E_{j}^{n+1}+E_{j}^{n}\right) \cdot \frac{F\left(\left|E_{j}^{n+1}\right|^{2}\right)-F\left(\left|E_{j}^{n}\right|^{2}\right)}{\left|E_{j}^{n+1}\right|^{2}-\left|E_{j}^{n}\right|^{2}}, \quad 1 \leq j \leq J, \\
\left(N_{j}^{n}\right)_{\bar{t} \bar{t}}-(1-2 \theta)\left(N_{j}^{n}\right)_{x \bar{x}}-\theta\left(\left(N_{j}^{n+1}\right)_{x \bar{x}}+\left(N_{j}^{n-1}\right)_{x \bar{x}}\right) \\
=\left(F\left(\left|E_{j}^{n}\right|^{2}\right)\right)_{x \bar{x}}, \quad 0 \leq \theta \leq \frac{1}{2} .
\end{gathered}
$$

The initial data and boundary conditions are approximated as

$$
\begin{aligned}
& E_{j}^{0}=E^{0}\left(x_{j}\right), \quad N_{j}^{0}=N^{0}\left(x_{j}\right), \\
& N_{j}^{1}=N_{j}^{0}+\tau N^{\prime}\left(x_{j}\right) \quad \text { or } \\
& N_{j}^{1}=N_{j}^{0}+\tau N^{1}\left(x_{j}\right)+\frac{\tau^{2}}{2}\left[\left(N_{j}^{0}\right)_{x \bar{x}}+\left(F\left(\left|E_{j}^{0}\right|^{2}\right)\right)_{x \bar{x}}\right], \\
& E_{0}^{n}=E_{J}^{n}=0, \quad N_{0}^{n}=N_{J}^{n}=0, \quad u_{0}^{n+\frac{1}{2}}=u_{J}^{n+\frac{1}{2}}=0,
\end{aligned}
$$

where the difference scheme (2.2) is used to approximate $N_{t t}^{0}$ in (2.4). We also define $\left\{u_{j}^{n+\frac{1}{2}}\right\}$, as R. T. Glassey did in [9], by

$$
\left(u_{j}^{n+\frac{1}{2}}\right)_{x \bar{x}}=\left(N_{j}^{n}\right)_{t}, \quad j=1,2, \ldots, J-1 .
$$

We note that the equations (2.1) are implicit, and a tridiagonal system of equations is involved. For $\theta=\frac{1}{2}$, the equations (2.2) are also implicit, and 
another tridiagonal system of equations needs to be solved. However, when $\theta=0$, then the scheme (2.2) for $N$ is explicit, and no tridiagonal system needs to be solved.

Theorem 1. The difference problem (2.1)-(2.6) possesses the following invariants:

$$
\left\|E^{n}\right\|_{2}^{2}=\text { Const }
$$

and

$$
\begin{aligned}
H_{h}^{n+\frac{1}{2}}= & \left\|E_{x}^{n+1}\right\|_{2}^{2}+\left\|E_{x}^{n}\right\|_{2}^{2}+\left\|u_{x}^{n+\frac{1}{2}}\right\|_{2}^{2}+(1-2 \theta) h \sum_{j=1}^{J} N_{j}^{n+1} N_{j}^{n} \\
& +\theta\left(\left\|N^{n+1}\right\|_{2}^{2}+\left\|N_{j}^{n}\right\|_{2}^{2}\right)+\frac{1}{2} h \sum_{j=1}^{J}\left[F\left(\left(\left|E_{j}^{n+1}\right|^{2}\right)+F\left(\left|E_{j}^{n}\right|^{2}\right)\right)\left(N_{j}^{n+1}+N_{j}^{n}\right)\right] \\
= & \text { Const. }
\end{aligned}
$$

Proof. Computing the inner product of (2.1) with $\left(E_{j}^{n+1}+E_{j}^{n}\right)$ implies

$$
\begin{aligned}
& i\left(\left(E_{j}^{n+1}\right)_{\bar{t}}, E_{j}^{n+1}+E_{j}^{n}\right)+\frac{1}{2}\left(\left(E_{j}^{n+1}\right)_{x \bar{x}}+\left(E_{j}^{n}\right)_{x \bar{x}}, E_{j}^{n+1}+E_{j}^{n}\right) \\
& \quad=\frac{1}{4}\left(\left(N_{j}^{n+1}+N_{j}^{n}\right)\left(E_{j}^{n+1}+E_{j}^{n}\right) \frac{F\left(\left|E_{j}^{n+1}\right|^{2}\right)-F\left(\left|E_{j}^{n}\right|^{2}\right)}{\left|E_{j}^{n+1}\right|^{2}-\left|E_{j}^{n}\right|^{2}}, E_{j}^{n+1}+E_{j}^{n}\right),
\end{aligned}
$$

where

$$
\begin{aligned}
& \left(\left(E_{j}^{n+1}\right)_{\bar{t}}, E_{j}^{n+1}+E_{j}^{n}\right) \\
& \quad=\frac{1}{\tau}\left(E_{j}^{n+1}-E_{j}^{n}, E_{j}^{n+1}+E_{j}^{n}\right) \\
& \quad=\frac{1}{\tau}\left(\left\|E^{n+1}\right\|_{2}^{2}-\left\|E^{n}\right\|_{2}^{2}\right)+h \sum_{j=1}^{J} E_{j}^{n+1} \cdot \overline{E_{j}^{n}}-h \sum_{j=1}^{J} E_{j}^{n} \cdot \overline{E_{j}^{n+1}}, \\
& \quad-\left(\left(E_{j}^{n+1}\right)_{x \bar{x}}+\left(E_{j}^{n}\right)_{x \bar{x}}, E_{j}^{n+1}+E_{j}^{n}\right) \\
& \quad=\left(\left(E_{j}^{n+1}\right)_{x}+\left(E_{j}^{n}\right)_{x},\left(E_{j}^{n+1}\right)_{x}+\left(E_{j}^{n}\right)_{x}\right) \\
& \left.=\left\|E_{x}^{n+1}\right\|_{2}^{2}+\left\|E_{x}^{n}\right\|_{2}^{2}+h \sum_{j=1}^{J}\left(E_{j}^{n+1}\right)_{x} \cdot\left(\overline{E_{j}^{n}}\right)_{x}+h \sum_{j=1}^{J}\left(E_{j}^{n}\right)_{x} \cdot \overline{\left(E_{j}^{n+1}\right.}\right)_{x}, \\
& \left(\left(N_{j}^{n+1}+N_{j}^{n}\right)\left(E_{j}^{n+1}+E_{j}^{n}\right) \frac{F\left(\left|E_{j}^{n+1}\right|^{2}\right)-F\left(\left|E_{j}^{n}\right|^{2}\right)}{\left|E_{j}^{n+1}\right|^{2}-\left|E_{j}^{n}\right|^{2}}, E_{j}^{n+1}+E_{j}^{n}\right) \\
& =h \sum_{j=1}^{J}\left(N_{j}^{n+1}+N_{j}^{n}\right) \frac{F\left(\left|E_{j}^{n+1}\right|^{2}\right)-F\left(\left|E_{j}^{n}\right|^{2}\right)}{\left|E_{j}^{n+1}\right|^{2}-\left|E_{j}^{n}\right|^{2}} \cdot\left|E_{j}^{n+1}+E_{j}^{n}\right|^{2} .
\end{aligned}
$$

Thus, we take the imaginary part of (2.7) and use the formulae derived above to get

$$
\frac{1}{\tau}\left(\left\|E^{n+1}\right\|_{2}^{2}-\left\|E^{n}\right\|_{2}^{2}\right)=0,
$$

i.e.,

$$
\left\|E^{n}\right\|_{2}^{2}=\left\|E^{0}\right\|_{2}^{2}=\text { Const. }
$$


Computing the inner product of $(2.1)$ with $\tau\left(E_{j}^{n+1}\right)_{\bar{t}}$ and taking the real part, we have

$$
-\frac{1}{2}\left(\left\|E_{x}^{n+1}\right\|_{2}^{2}-\left\|E_{x}^{n}\right\|_{2}^{2}\right)=\frac{1}{4} h \sum_{j=1}^{J}\left(N_{j}^{n+1}+N_{j}^{n}\right)\left[F\left(\left|E_{j}^{n+1}\right|^{2}\right)-F\left(\left|E_{j}^{n}\right|^{2}\right)\right]
$$

Next, we compute the inner product of (2.2) with $\left(u_{j}^{n+\frac{1}{2}}+u_{j}^{n-\frac{1}{2}}\right)$, and by using (2.6) we obtain

$$
\begin{aligned}
\left(\left(N_{j}^{n}\right)_{t \bar{t}}, u_{j}^{n+\frac{1}{2}}+u_{j}^{n-\frac{1}{2}}\right)-(1-2 \theta)\left(N_{j}^{n},\left(N_{j}^{n}\right)_{t}+\left(N_{j}^{n-1}\right)_{t}\right) & \\
& -\theta\left(N_{j}^{n+1}+N_{j}^{n-1},\left(N_{j}^{n}\right)_{t}+\left(N_{j}^{n-1}\right)_{t}\right) \\
= & \left(F\left(\left|E_{j}^{n}\right|^{2}\right),\left(N_{j}^{n}\right)_{t}+\left(N_{j}^{n-1}\right)_{t}\right)
\end{aligned}
$$

which is equivalent to

(2.10)

$$
\begin{gathered}
\left\|u_{x}^{n+\frac{1}{2}}\right\|_{2}^{2}+\left\|u_{x}^{n-\frac{1}{2}}\right\|_{2}^{2}+(1-2 \theta) h \sum_{j=1}^{J} N_{j}^{n+1} \cdot N_{j}^{n}-(1-2 \theta) h \sum_{j=1}^{J} N_{j}^{n} \cdot N_{j}^{n-1} \\
+\theta\left(\left\|N^{n+1}\right\|_{2}^{2}-\left\|N^{n-1}\right\|_{2}^{2}\right)+h \sum_{j=1}^{J} F\left(\left|E_{j}^{n}\right|^{2}\right)\left(N_{j}^{n+1}-N_{j}^{n-1}\right)=0
\end{gathered}
$$

where (2.6) is used to reduce the first term.

It follows from (2.9) that

$$
\begin{aligned}
-\left\|E_{x}^{n+1}\right\|_{2}^{2}+\left\|E_{x}^{n-1}\right\|_{2}^{2}= & \frac{1}{2} h \sum_{j=1}^{J}\left(F\left(\left|E_{j}^{n+1}\right|^{2}\right)-F\left(\left|E_{j}^{n}\right|^{2}\right)\right)\left(N_{j}^{n+1}+N_{j}^{n}\right) \\
& -\frac{1}{2} h \sum_{j=1}^{J}\left(F\left(\left|E_{j}^{n}\right|^{2}\right)+F\left(\left|E_{j}^{n-1}\right|^{2}\right)\right)\left(N_{j}^{n}+N_{j}^{n-1}\right) \\
& -h \sum_{j=1}^{J}\left(F\left(\left|E_{j}^{n}\right|^{2}\right)\right)\left(N_{j}^{n+1}-N_{j}^{n-1}\right) .
\end{aligned}
$$

Combining (2.10) and (2.11) yields

$$
H_{h}^{n+\frac{1}{2}}=H_{h}^{n-\frac{1}{2}}=\text { Const. }
$$

Theorem 2. Assume $E(x, t) \in C^{5}, N(x, t) \in C^{5}$ for the solution of problem (1.5)-(1.11) and $f(s) \in C^{2}\left(R^{+}\right)$. Then the difference scheme (2.1)-(2.2) possesses truncation errors of order $O\left(h^{2}+\tau^{2}\right)$.

Proof. By Taylor's expansion, we have

$$
\begin{gathered}
E(j, n+1)+E(j, n)=\left.\left[2 E+\frac{\tau^{2}}{4} E_{t t}+O\left(\tau^{3}\right)\right]\right|_{x=x_{j}, t=t^{n+\frac{1}{2}}}, \\
(E(j, n+1))_{\bar{t}}=\left.\left[E_{t}+\frac{\tau^{2}}{24} E_{t t t}+O\left(\tau^{3}\right)\right]\right|_{x=x_{j}, t=t^{n+\frac{1}{2}}},
\end{gathered}
$$




$$
(N(j, n))_{x \bar{x}}=\left.\left[N_{x x}+\frac{h^{2}}{12} N_{x x x x}+O\left(h^{3}\right)\right]\right|_{x=x_{j}, t=t^{n}},
$$

and

$$
\begin{aligned}
& \frac{F\left(|E(j, n+1)|^{2}\right)-F\left(|E(j, n)|^{2}\right)}{|E(j, n+1)|^{2}-|E(j, n)|^{2}} \\
& =\left\{\left[F\left(\left|E\left(j, n+\frac{1}{2}\right)\right|^{2}\right)+F^{\prime}\left(\left|E\left(j, n+\frac{1}{2}\right)\right|^{2}\right)\left(|E(j, n+1)|^{2}-\left|E\left(j, n+\frac{1}{2}\right)\right|^{2}\right)\right.\right. \\
& +\frac{1}{2} F^{\prime \prime}\left(\left|E\left(j, n+\frac{1}{2}\right)\right|^{2}\right)\left(|E(j, n+1)|^{2}-\left|E\left(j, n+\frac{1}{2}\right)\right|^{2}\right)^{2} \\
& \left.+\frac{1}{3 !} F^{\prime \prime \prime}\left(\left|E\left(j, n+\frac{1}{2}\right)\right|^{2}\right)\left(|E(j, n+1)|^{2}-\left|E\left(j, n+\frac{1}{2}\right)\right|^{2}\right)^{3}+\cdots\right] \\
& -\left[F\left(\left|E\left(j, n+\frac{1}{2}\right)\right|^{2}\right)+F^{\prime}\left(\left|E\left(j, n+\frac{1}{2}\right)\right|^{2}\right)\left(|E(j, n)|^{2}-\left|E\left(j, n+\frac{1}{2}\right)\right|^{2}\right)\right. \\
& +\frac{1}{2} F^{\prime \prime}\left(\left|E\left(j, n+\frac{1}{2}\right)\right|^{2}\right)\left(|E(j, n)|^{2}-\left|E\left(j, n+\frac{1}{2}\right)\right|^{2}\right)^{2} \\
& \left.\left.+\frac{1}{3 !} F^{\prime \prime \prime}\left(\left|E\left(j, n+\frac{1}{2}\right)\right|^{2}\right)\left(|E(j, n)|^{2}-\left|E\left(j, n+\frac{1}{2}\right)\right|^{2}\right)^{3}+\cdots\right]\right\} / \\
& {\left[|E(j, n+1)|^{2}-|E(j, n)|^{2}\right]} \\
& =F^{\prime}\left(\left|E\left(j, n+\frac{1}{2}\right)\right|^{2}\right)+\frac{1}{2} F^{\prime \prime}\left(\left|E\left(j, n+\frac{1}{2}\right)\right|^{2}\right) \\
& \cdot\left(|E(j, n+1)|^{2}+|E(j, n)|^{2}-2\left|E\left(j, n+\frac{1}{2}\right)\right|^{2}\right)+\frac{1}{6} F^{\prime \prime \prime}\left(\left|E\left(j, n+\frac{1}{2}\right)\right|^{2}\right) \\
& \cdot\left[\left(|E(j, n+1)|^{2}-\left|E\left(j, n+\frac{1}{2}\right)\right|^{2}\right)^{2}\right. \\
& +\left(|E(j, n+1)|^{2}-\left|E\left(j, n+\frac{1}{2}\right)\right|^{2}\right) \cdot\left(|E(j, n)|^{2}-\left|E\left(j, n+\frac{1}{2}\right)\right|^{2}\right) \\
& \left.+\left(|E(j, n)|^{2}-\left|E\left(j, n+\frac{1}{2}\right)\right|^{2}\right)^{2}\right]+O\left(\tau^{3}\right) \\
& =F^{\prime}\left(\left|E\left(j, n+\frac{1}{2}\right)\right|^{2}\right)+\frac{1}{8} F^{\prime \prime}\left(\left|E\left(j, n+\frac{1}{2}\right)\right|^{2}\right) \cdot \tau^{2} \cdot\left(\left|E\left(j, n+\frac{1}{2}\right)\right|^{2}\right)_{t t} \\
& +\frac{1}{6} F^{\prime \prime \prime}\left(\left|E\left(j, n+\frac{1}{2}\right)\right|^{2}\right) \\
& \cdot\left(|E(j, n+1)|^{4}+|E(j, n)|^{4}+3\left|E\left(j, n+\frac{1}{2}\right)\right|^{4}\right. \\
& +|E(j, n+1)|^{2} \cdot|E(j, n)|^{2}-3|E(j, n+1)|^{2}\left|E\left(j, n+\frac{1}{2}\right)\right|^{2} \\
& \left.-3|E(j, n)|^{2} \cdot\left|E\left(j, n+\frac{1}{2}\right)\right|^{2}\right)+O\left(\tau^{3}\right) \\
& =f\left(\left|E\left(j, n+\frac{1}{2}\right)\right|^{2}\right)+\frac{\tau^{3}}{8} f^{\prime}\left(\left|E\left(j, n+\frac{1}{2}\right)\right|^{2}\right) \cdot\left(\left|E\left(j, n+\frac{1}{2}\right)\right|^{2}\right)_{t t} \\
& +\frac{1}{6} f^{\prime \prime}\left(\left|E\left(j, n+\frac{1}{2}\right)\right|^{2}\right) \cdot \frac{\tau^{2}}{2}\left[\left(\left|E\left(j, n+\frac{1}{2}\right)\right|^{2}\right)_{t}\right]^{2}+O\left(\tau^{3}\right) .
\end{aligned}
$$

Using equalities derived above, we obtain from the difference schemes (2.1) and (2.2)

(2.12)

$$
\begin{aligned}
i E_{t}+E_{x x}= & N f\left(|E|^{2}\right) \cdot E \\
& +\left[-\frac{i \tau^{2}}{24} E_{t t t}-\frac{\tau^{2}}{8} E_{x x t t}-\frac{h^{2}}{12} E_{x x x x}\right. \\
& \quad+\frac{\tau^{2}}{8} E f\left(|E|^{2}\right) N_{t t}+\frac{\tau^{2}}{8} N f\left(|E|^{2}\right) E_{t t}+\frac{\tau^{2}}{8} N E f^{\prime}\left(|E|^{2}\right)\left(|E|^{2}\right)_{t t} \\
& \left.+\frac{\tau^{2}}{12} N E f^{\prime \prime}\left(|E|^{2}\right)\left(\left(|E|^{2}\right)_{t}\right)^{2}\right] \\
& +O\left(h^{3}+\tau^{3}\right)
\end{aligned}
$$


and

$$
\begin{aligned}
N_{t t}-N_{x x}= & \left(F\left(|E|^{2}\right)\right)_{x x} \\
& +\left[-\frac{\tau^{2}}{12} N_{t t t t}+\theta \cdot \tau^{2} N_{x x t t}+\frac{h^{2}}{12} N_{x x x x}+\frac{h^{4}}{12}\left(F\left(|E|^{2}\right)\right)_{x x x x}\right] \\
& +O\left(h^{3}+\tau^{3}\right)
\end{aligned}
$$

Thus, the truncation errors are $O\left(h^{2}+\tau^{2}\right)$.

In [5], we have used the conservative scheme to compute solitary waves and the interaction of two colliding solitary waves for the Zakharov equations (1.1) and (1.2). Numerical experiments demonstrate that the semiexplicit scheme with $\theta=0$ is more efficient and accurate than the implicit scheme with $\theta=\frac{1}{2}$. For example, if we require that computational errors be less than 0.1 during the entire time period of the solitary wave, then the step sizes $h=\tau=0.25$ can be taken for the scheme with $\theta=0$, but the step sizes $h=\tau=0.1$ are needed for the scheme with $\theta=\frac{1}{2}$. To achieve the same accuracy, the scheme with $\theta=\frac{1}{2}$ takes a longer CPU time than the scheme with $\theta=0$, and the ratio of the CPU times used by the two schemes is

$$
R_{t}=\frac{534.94}{78.26} \approx 6.8
$$

\section{CONVERGENCE OF THE DIFFERENCE SCHEME}

In this section, we consider the convergence of the difference scheme (2.1)(2.6).

We define the errors by

$$
e_{j}^{n}=E(j, n)-E_{j}^{n} \quad \text { and } \quad \eta_{j}^{n}=N(j, n)-N_{j}^{n} .
$$

Let

$$
\left(U_{j}^{n+\frac{1}{2}}\right)_{x \bar{x}}=\left(\eta_{j}^{n+1}\right)_{\bar{t}}, \quad U_{0}^{n+\frac{1}{2}}=U_{J}^{n+\frac{1}{2}}=0 .
$$

Then the error equations are obtained as follows:

$$
\begin{aligned}
& i\left(e_{j}^{n+1}\right)_{\bar{t}}+\frac{1}{2}\left[\left(e_{j}^{n+1}\right)_{x \bar{x}}+\left(e_{j}^{n}\right)_{x \bar{x}}\right] \\
& =R^{E}+\frac{1}{4}[N(j, n)+N(j, n+1)] \frac{F\left(|E(j, n+1)|^{2}\right)-F\left(|E(j, n)|^{2}\right)}{|E(j, n+1)|^{2}-|E(j, n)|^{2}} \\
& \cdot[E(j, n+1)+E(j, n)] \\
& -\frac{1}{4}\left(N_{j}^{n}+N_{j}^{n+1}\right) \frac{F\left(\left|E_{j}^{n+1}\right|^{2}\right)-F\left(\left|E_{j}^{n}\right|^{2}\right)}{\left|E_{j}^{n+1}\right|^{2}-\left|E_{j}^{n}\right|^{2}}\left(E_{j}^{n+1}+E_{j}^{n}\right) \\
& \left(\eta_{j}^{n}\right)_{\bar{t}}-(1-2 \theta)\left(\eta_{j}^{n}\right)_{x \bar{x}}-\theta\left[\left(\eta_{j}^{n-1}\right)_{x \bar{x}}+\left(\eta_{j}^{n-1}\right)_{x \bar{x}}\right] \\
& =R^{N}+\left[F(|E(j, n)|)^{2}-F\left(\left|E_{j}^{n}\right|^{2}\right)\right]_{x \bar{x}},
\end{aligned}
$$


where

$$
\begin{aligned}
R^{E}= & -\frac{i \tau^{2}}{24} E_{t t t}-\frac{\tau^{2}}{8} E_{x x t t}-\frac{h^{2}}{12} E_{x x x x} \\
& +\frac{\tau^{2}}{8} E f\left(|E|^{2}\right) N_{t t}+\frac{\tau^{2}}{8} N f\left(|E|^{2}\right) E_{t t}+\frac{\tau^{2}}{8} N E f^{\prime}\left(|E|^{2}\right)\left(|E|^{2}\right)_{t t} \\
& \left.\quad+\frac{\tau^{2}}{12} N E f^{\prime \prime}\left(|E|^{2}\right)\left(\left(|E|^{2}\right)_{t}\right)^{2}\right]\left.\right|_{x=x_{j}, t=t^{n+\frac{1}{2}}} \\
+ & O\left(h^{3}+\tau^{3}\right), \\
R^{N=} & {\left.\left[-\frac{\tau^{2}}{12} N_{t t t t}+\theta \cdot \tau^{2} N_{x x t t}+\frac{h^{2}}{12} N_{x x x x}+\frac{h^{4}}{12}\left(F\left(|E|^{2}\right)\right)_{x x x x}\right]\right|_{x=x_{j}, t=t^{n}} } \\
& +O\left(h^{3}+\tau^{3}\right),
\end{aligned}
$$

in view of the formulae (2.12) and (2.13).

Lemma 1 (Sobolev estimate [8]). Suppose $W \in L_{q}\left(R^{n}\right), D^{m} W \in L_{r}\left(R^{n}\right), 1 \leq$ $q, r<\infty$. Then for $0 \leq j \leq m, \frac{j}{m} \leq \alpha \leq 1$, we have

$$
\left\|D^{j} W\right\|_{L_{p}} \leq C\left\|D^{m} W\right\|_{L_{r}}^{\alpha} \cdot\|W\|_{L_{q}}^{1-\alpha}
$$

where $\frac{1}{p}=\frac{j}{n}+\alpha\left(\frac{1}{r}-\frac{m}{n}\right)+(1-\alpha) \frac{1}{q}$.

Lemma 2. Let $r=\frac{\tau}{h}<\sqrt{\frac{1}{1-2 \theta}}, 0 \leq \theta \leq \frac{1}{2}$. If we define $C_{1}=\frac{2+(1-2 \theta) r^{2}}{2-(1-2 \theta) r^{2}}$, then the following inequality holds:

$$
R_{\tau} \leq C_{1} Q_{\tau}
$$

where

$$
\begin{gathered}
R_{\tau}=\left\|u_{x}^{n+\frac{1}{2}}\right\|_{2}^{2}+\frac{1}{2}(1-2 \theta)\left(\left\|N^{n+1}\right\|_{2}^{2}+\left\|N^{n}\right\|_{2}^{2}\right), \\
Q_{\tau}=\left\|u_{x}^{n+\frac{1}{2}}\right\|_{2}^{2}+(1-2 \theta) h \sum_{j=1}^{J} N_{j}^{n+1} \cdot N_{j}^{n} .
\end{gathered}
$$

Proof of Lemma 2. Let $\left(W_{j}^{n}\right)_{t}=u_{j}^{n+\frac{1}{2}}$ and $\left(W_{j}^{0}\right)_{x \bar{x}}=N_{j}^{0} ;$ then $W_{0}^{n}=W_{J}^{n}=0$ and $N_{j}^{n}=\left(W_{j}^{n}\right)_{x \bar{x}}$. Thus, we have

$$
\begin{aligned}
& Q_{\tau}=h \sum_{j=1}^{J}\left[\left(W_{j}^{n}\right)_{x t}\right]^{2}+(1-2 \theta) h \sum_{j=1}^{J}\left(W_{j}^{n+1}\right)_{x \bar{x}} \cdot\left(W_{j}^{n}\right)_{x \bar{x}}, \\
& R_{\tau}=h \sum_{j=1}^{J}\left[\left(W_{j}^{n}\right)_{x t}\right]^{2}+\frac{1}{2}(1-2 \theta) h \sum_{j=1}^{J}\left[\left(N_{j}^{n+1}\right)^{2}+\left(N_{j}^{n}\right)^{2}\right] .
\end{aligned}
$$

We use the following notation:

$$
\begin{aligned}
& D W_{j}^{n} \equiv\left(W_{j}^{n}\right)_{x}, \quad D^{2} W_{j}^{n} \equiv\left(W_{j}^{n}\right)_{x \bar{x}}, \\
& Q_{D} \equiv\left[\begin{array}{cc}
-\tau^{-2} D^{2}, & \tau^{-2} D^{2}+\frac{1}{2}(1-2 \theta) D^{4} \\
\tau^{-2} D^{2} & +\frac{1}{2}(1-2 \theta) D^{4},
\end{array}\right]
\end{aligned}
$$

and

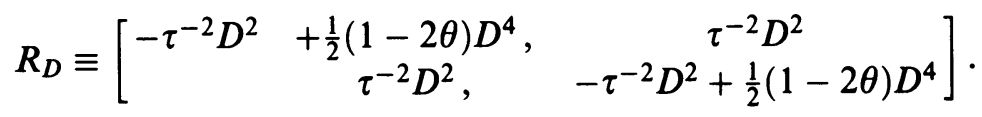


It is easily verified that

$$
\begin{aligned}
& Q_{\tau}=h \sum_{j=1}^{J}\left(W_{j}^{n+1}, W_{j}^{n}\right) \\
& \cdot\left[\begin{array}{ccc}
-\tau^{-2} D^{2}, & \tau^{-2} D^{2}+\frac{1}{2}(1-2 \theta) D^{4} & \\
\tau^{-2} D^{2} & +\frac{1}{2}(1-2 \theta) D^{4}, & -\tau^{-2} D^{2}
\end{array}\right]\left(\begin{array}{c}
W_{j}^{n+1} \\
W_{j}^{n}
\end{array}\right) \\
& =h \sum_{j=1}^{J}\left(W_{j}^{n+1}, W_{j}^{n}\right) \cdot Q_{D} \cdot\left(\begin{array}{c}
W_{j}^{n+1} \\
W_{j}^{n}
\end{array}\right) \text {, }
\end{aligned}
$$

and

$$
R_{\tau}=h \sum_{j=1}^{J}\left(W_{j}^{n+1}, W_{j}^{n}\right) \cdot R_{D} \cdot\left(\begin{array}{c}
W_{j}^{n+1} \\
W_{j}^{n}
\end{array}\right) .
$$

Assume that $\left(Y_{1}, Y_{2}\right)$ is an eigenfunction associated with an eigenvalue $\lambda$ of $Q_{D}$; then

$$
\begin{aligned}
-\tau^{-2} D^{2} Y_{1}+\tau^{-2} D^{2} Y_{2}+\frac{1}{2}(1-2 \theta) D^{4} Y_{2} & =\lambda Y_{1}, \\
\tau^{-2} D^{2} Y_{1}+\frac{1}{2}(1-2 \theta) D^{4} Y_{1}-\tau^{-2} D^{2} Y_{2} & =\lambda Y_{2}
\end{aligned}
$$

By adding and subtracting these equations, we obtain

$$
\begin{gathered}
\frac{1}{2}(1-2 \theta) D^{4}\left(Y_{1}+Y_{2}\right)=\lambda\left(Y_{1}+Y_{2}\right) \\
-2 \tau^{-2} D^{2}\left(Y_{1}-Y_{2}\right)-\frac{1}{2}(1-2 \theta) D^{4}\left(Y_{1}-Y_{2}\right)=\lambda\left(Y_{1}-Y_{2}\right) .
\end{gathered}
$$

If we look for an eigenfunction with $Y_{1}=Y_{2}=Y$, then (3.8) always holds and (3.7) implies that $Y$ is an eigenfunction of the operator $\frac{1}{2}(1-2 \theta) D^{4}$ with eigenvalue $\frac{1}{2}(1-2 \theta) \mu_{4}$, where $\mu_{4}$ is the eigenvalue of $D^{4}$. This provides $J$ eigenvalues of $Q_{D}$. On the other hand, if we seek an eigenfunction with $Y_{1}=-Y_{2}=Y$, then (3.7) holds and (3.8) implies that the eigenvalue $\lambda$ is of the form $-2 \tau^{2} \mu_{2}-\frac{1}{2}(1-2 \theta) \mu_{4}$ with $\mu_{2}$ an eigenvalue of $D^{2}$.

For an eigenvalue of $R_{D}$, we have

$$
\begin{aligned}
-\tau^{-2} D^{2} Y_{1}+\frac{1}{2}(1-2 \theta) D^{4} Y_{1}+\tau^{-2} D^{2} Y_{2} & =\lambda Y_{1}, \\
\tau^{-2} D^{2} Y_{1}-\tau^{-2} D^{2} Y_{2}+\frac{1}{2}(1-2 \theta) D^{4} Y_{2} & =\lambda Y_{2}
\end{aligned}
$$

A similar argument yields that the eigenvalues and eigenfunctions of $R_{D}$ are

$$
\left\{\frac{1}{2}(1-2 \theta) \mu_{4},(Y, Y)\right\},\left\{-2 \tau^{-2} \mu_{2}+\frac{1}{2}(1-2 \theta) \mu_{4},(Y,-Y)\right\} \text {. }
$$

Since $R_{D}$ and $Q_{D}$ have a common set of eigenfunctions, the inequality $R_{\tau} \leq$ $C Q_{\tau}$ is equivalent to

$$
\lambda\left(R_{D}\right) \leq C \lambda\left(Q_{D}\right)
$$

for the corresponding eigenvalues.

It follows from Fourier analysis that the eigenvalues of the operators $D^{2}$ and $D^{4}$ are

$$
\mu_{2}=2 h^{-2}\left(\cos \frac{2 \pi j h}{x_{R}-x_{L}}-1\right) \quad \text { and } \mu_{4}=\mu_{2}^{2}, \quad j=1,2, \ldots, J .
$$


Thus, we have

$$
\begin{aligned}
& \lambda_{j}^{R}=-2 \tau^{-2} \cdot 2 h^{-2}\left(\cos \frac{2 \pi j h}{x_{R}-x_{L}}-1\right)+(1-2 \theta) 2 h^{-4}\left(\cos \frac{2 \pi j h}{x_{R}-x_{L}}-1\right)^{2}, \\
& \lambda_{j}^{Q}=-2 \tau^{-2} \cdot 2 h^{-2}\left(\cos \frac{2 \pi j h}{x_{R}-x_{L}}-1\right)+(1-2 \theta) 2 h^{-4}\left(\cos \frac{2 \pi j h}{x_{R}-x_{L}}-1\right)^{2} .
\end{aligned}
$$

Substituting these eigenvalues into (3.9) yields

$$
\begin{aligned}
4 \tau^{-2} & \cdot h^{-2}\left(1-\cos \frac{2 \pi j h}{x_{R}-x_{L}}\right)+2(1-2 \theta) h^{-4}\left(1-\cos \frac{2 \pi j h}{x_{R}-x_{L}}\right)^{2} \\
\leq & \leq\left[4 \tau^{-2} h^{-2}\left(1-\cos \frac{2 \pi j h}{x_{R}-x_{L}}\right)-2(1-2 \theta) h^{-4}\left(1-\cos \frac{2 \pi j h}{x_{R}-x_{L}}\right)^{2}\right],
\end{aligned}
$$

i.e.,

$$
2+(1-2 \theta) r^{2}\left(1-\cos \frac{2 \pi j h}{x_{R}-x_{L}}\right) \leq\left[2-(1-2 \theta) r^{2}\left(1-\cos \frac{2 \pi j h}{x_{R}-x_{L}}\right)\right] .
$$

The inequality $(3.10)$ holds with $C_{1}=\frac{2+(1-2 \theta) r^{2}}{2-(1-2 \theta) r^{2}}$, provided $r<\sqrt{\frac{1}{1-2 \theta}}$. This completes the proof.

Lemma 3. Assume $E^{0}(x) \in H_{0}^{1}, N^{0}(x) \in L_{2}, N^{1}(x) \in L_{2} ;|F(s)| \leq C_{1} s^{\alpha}+$ $C_{2}, 0 \leq \alpha<\frac{3}{2}$, for $s \geq 0$, and $r=\frac{\tau}{h}<\sqrt{\frac{1}{1-2 \theta}}, 0 \leq \theta \leq \frac{1}{2}$. Then we have the estimates

$$
\begin{gathered}
\left\|E^{n}\right\|_{2} \leq C, \quad\left\|E_{x}^{n}\right\|_{2} \leq C, \quad\left\|E^{n}\right\|_{\infty} \leq C, \\
\left\|N^{n}\right\|_{2} \leq C, \quad\left\|u_{x}^{n+\frac{1}{2}}\right\|_{2} \leq C, \quad\left\|u^{n+\frac{1}{2}}\right\|_{\infty} \leq C, \quad 0 \leq n \leq \frac{T}{\tau} .
\end{gathered}
$$

Proof. It follows from Theorem 1 that

$$
\left\|E^{n}\right\|_{2} \leq C
$$

and

$$
\begin{aligned}
& \left\|E_{x}^{n+1}\right\|_{2}^{2}+\left\|E_{x}^{n}\right\|_{2}^{2}+\left\|u_{x}^{n+\frac{1}{2}}\right\|_{2}^{2}+(1-2 \theta) h \sum_{j=1}^{J} N_{j}^{n+1} \cdot N_{j}^{n} \\
& \quad+\theta\left(\left\|N^{n+1}\right\|_{2}^{2}+\left\|N^{n}\right\|_{2}^{2}\right)+\frac{1}{2} h \sum_{j=1}^{J}\left[F\left(\left|E_{j}^{n+1}\right|^{2}\right)+F\left(\left|E_{j}^{n}\right|^{2}\right)\right]\left(N_{j}^{n+1}+N_{j}^{n}\right) \\
& \quad \text { Const. }
\end{aligned}
$$

Using Lemma 2, we have

$$
\begin{gathered}
\left\|E_{x}^{n+1}\right\|_{2}^{2}+\left\|E_{x}^{n}\right\|_{2}^{2}+\frac{1}{C_{1}}\left\|u_{x}^{n+\frac{1}{2}}\right\|_{2}^{2}+\left(\frac{1}{2} \frac{1-2 \theta}{C_{1}}+\theta\right)\left(\left\|N^{n+1}\right\|_{2}^{2}-\left\|N^{n}\right\|_{2}^{2}\right) \\
+\frac{1}{2} h \sum_{j=1}^{J}\left[F\left(\left|E_{j}^{n+1}\right|^{2}\right)+F\left(\left|E_{j}^{n}\right|^{2}\right)\right]\left(N_{j}^{n+1}+N_{j}^{n}\right) \leq C .
\end{gathered}
$$


The last term $\mathscr{L}$ on the left of the inequality (3.11) is estimated by

$$
\begin{aligned}
|\mathscr{L}| \leq & \frac{1}{2} h \sum_{j=1}^{J} \mid\left[F\left(\left|E_{j}^{n+1}\right|^{2}\right) \cdot N_{j}^{n+1}+F\left(\left|E_{j}^{n}\right|^{2}\right) \cdot N_{j}^{n+1}\right. \\
& \left.+F\left(\left|E_{j}^{n+1}\right|^{2}\right) N_{j}^{n}+F\left(\left|E_{j}^{n}\right|^{2}\right) N_{j}^{n}\right] \mid \\
\leq & \frac{\varepsilon_{1}}{2} h \sum_{j=1}^{J}\left[\left(N_{j}^{n+1}\right)^{2}+\left(N_{j}^{n}\right)^{2}\right] \\
& +\frac{1}{2 \varepsilon_{1}} h \sum_{j=1}^{J}\left[\left(F\left(\left|E_{j}^{n+1}\right|^{2}\right)\right)^{2}+\left(F\left(\left|E_{j}^{n}\right|^{2}\right)\right)^{2}\right]
\end{aligned}
$$

for any $\varepsilon_{1}>0$. By Lemma 1 and the Interpolation Lemma [14], we get

$$
\begin{aligned}
& h \sum_{j=1}^{J}\left[\left(F\left(\left|E_{j}^{n+1}\right|^{2}\right)\right)^{2}+\left(F\left(\left|E_{j}^{n}\right|^{2}\right)\right)^{2}\right] \\
& \leq C h \sum_{j=1}^{J}\left(\left.\left|E_{j}^{n+1}\right|^{4 \alpha}|+| E_{j}^{n}\right|^{4 \alpha}+\left|E_{j}^{n+1}\right|^{2 \alpha}+\left|E_{j}^{n}\right|^{2 \alpha}\right)+C \\
& =C+C h \sum_{j=1}^{J}\left(\left|E_{j}^{n+1}\right|^{6-2 \delta}+\left|E_{j}^{n}\right|^{6-2 \delta}+\left|E_{j}^{n+1}\right|^{3-\delta}+\left|E_{j}^{n}\right|^{3-\delta}\right) \\
& \leq C+C\left[\left\|E_{x}^{n+1}\right\|_{2}^{2-\delta} \cdot\left\|E^{n+1}\right\|^{4-\delta}+\left\|E_{x}^{n}\right\|_{2}^{2-\delta} \cdot\left\|E^{n}\right\|_{2}^{4-\delta}\right. \\
& \left.\quad+\left\|E_{x}^{n+1}\right\|_{2}^{\frac{1}{2}-\frac{\delta}{2}} \cdot\left\|E^{n+1}\right\|_{2}^{\frac{5}{2}-\frac{\delta}{2}}+\left\|E_{x}^{n}\right\|^{\frac{1}{2}-\frac{\delta}{2}} \cdot\left\|E^{n}\right\|_{2}^{\frac{5}{2}-\frac{\delta}{2}}\right],
\end{aligned}
$$

where $\alpha=\frac{3}{2}-\frac{\delta}{2}, 3 \geq \delta>0$.

Using the inequality

$$
a \cdot b \leq \frac{1}{p}\left(\varepsilon_{2} a\right)^{p}+\frac{1}{p^{\prime}}\left(\frac{1}{\varepsilon_{2}} b\right)^{p^{\prime}}, \quad \frac{1}{p}+\frac{1}{p^{\prime}}=1, a, b, p, p^{\prime}, \varepsilon_{2}>0,
$$

we have

$$
\begin{aligned}
& h \sum_{j=1}^{J}\left[\left(F\left(\left|E_{j}^{n+1}\right|^{2}\right)\right)^{2}+\left(F\left(\left|E_{j}^{n}\right|^{2}\right)\right)^{2}\right] \\
& \quad \leq C+\varepsilon_{2}\left(\left\|E_{x}^{n+1}\right\|_{2}^{2}+\left\|E_{x}^{n}\right\|_{2}^{2}\right)+\frac{C}{\varepsilon_{2}} .
\end{aligned}
$$

Substituting (3.12) and (3.13) into (3.11) and choosing $\varepsilon_{1}=\varepsilon_{2}=\frac{1-2 \theta}{2 C_{1}}+\theta$, we get the following inequality:

$$
\begin{aligned}
& \frac{1}{2}\left\|E_{x}^{n+1}\right\|_{2}^{2}+\frac{1}{2}\left\|E_{x}^{n}\right\|_{2}^{2}+\frac{1}{C_{1}}\left\|u_{x}^{n+\frac{1}{2}}\right\|_{2}^{2} \\
& \quad+\frac{1}{2}\left(\frac{1-2 \theta}{2 C_{1}}+\theta\right)\left(\left\|N^{n+1}\right\|_{2}^{2}+\left\|N^{n}\right\|_{2}^{2}\right) \leq C,
\end{aligned}
$$

from which the following estimates are obtained:

$$
\left\|E_{x}^{n}\right\|_{2}^{2} \leq C, \quad\left\|N^{n}\right\|_{2}^{2} \leq C, \quad\left\|u_{x}^{n+\frac{1}{2}}\right\|_{2}^{2} \leq C .
$$


It follows from the discrete Sobolev inequality in one dimension that

$$
\left\|E^{n}\right\|_{\infty} \leq C, \quad\left\|u^{n+\frac{1}{2}}\right\|_{\infty} \leq C .
$$

The proofs of the following two lemmas are given in the Supplement section.

Lemma 4. Assume that the function $F(s)$ is analytic in $R^{+}, E^{0}(x) \in H_{0}^{1}, N^{0}(x)$ $\in L_{2}, N^{1}(x) \in L_{2} ; f(s) \in C^{\infty}\left(R^{+}\right),|F(s)| \leq C_{1} s^{\alpha}+C_{2}, 0 \leq \alpha<\frac{3}{2}$, for $s \geq 0$, and $r=\frac{\tau}{\hbar}<\sqrt{\frac{1}{1-2 \theta}}, 0 \leq \theta \leq \frac{1}{2}$. Suppose that the solution of problem (1.5)-(1.11) satisfies $E(x, t) \in C^{5}, N(x, t) \in C^{5}$. Then we have the following estimates:

$$
\begin{aligned}
& \left|P_{1}^{n+\frac{1}{2}}-P_{n}^{n+\frac{1}{2}}\right| \\
& \quad \leq C \tau\left(h^{2}+\tau^{2}\right)^{2}+C \tau\left(\left\|e^{n+1}\right\|_{2}^{2}+\left\|e^{n}\right\|_{2}^{2}+\left\|e_{x}^{n+1}\right\|_{2}^{2}+\left\|e_{x}^{n}\right\|_{2}^{2}+\left\|\eta^{n+1}\right\|_{2}^{2}+\left\|\eta^{n}\right\|_{2}^{2}\right),
\end{aligned}
$$

where

$$
\begin{aligned}
& P_{1}^{n+\frac{1}{2}}=\operatorname{Re}((N(j, n)+N(j, n+1)) \frac{F\left(|E(j, n+1)|^{2}\right)-F\left(|E(j, n)|^{2}\right)}{|E(j, n+1)|^{2}-|E(j, n)|^{2}} \\
& \cdot(E(j, n+1)+E(j, n)) \\
&\left.-\left(N_{j}^{n}+N_{j}^{n+1}\right) \frac{F\left(\left|E_{j}^{n+1}\right|^{2}\right)-F\left(\left|E_{j}^{n}\right|^{2}\right)}{\left|E_{j}^{n+1}\right|^{2}-\left|E_{j}^{n}\right|^{2}}\left(E_{j}^{n+1}+E_{j}^{n}\right), e_{j}^{n+1}-e_{j}^{n}\right), \\
& P_{2}^{n+\frac{1}{2}}=h \sum_{j=1}^{J}\left[F\left(|E(j, n+1)|^{2}\right)-F\left(|E(j, n)|^{2}\right)\right. \\
&\left.-F\left(\left|E_{j}^{n+1}\right|^{2}\right)+F\left(|E(j, n)|^{2}\right)\right]\left(\eta_{j}^{n+1}+\eta_{j}^{n}\right) .
\end{aligned}
$$

Lemma 5. Assume $E^{0}(x) \in H_{0}^{1}, N^{0}(x) \in L_{2}, N^{1}(x) \in L_{2} ; f(s) \in C^{\infty}\left(R^{+}\right)$, $|F(s)| \leq C_{1} s^{\alpha}+C_{2}, 0 \leq \alpha<\frac{3}{2}$, for $s \geq 0$, and $r=\frac{\tau}{h}<\sqrt{\frac{1}{1-2 \theta}}, 0 \leq \theta \leq$ $\frac{1}{2}$. Suppose that the solution of problem (1.5)-(1.11) satisfies $E(x, t) \in C^{5}$, $N(x, t) \in C^{5}$. Then we have the following estimates:

$$
\begin{aligned}
\left|\left(R^{E}, e_{j}^{n+1}-e_{j}^{n}\right)\right| \leq & C \tau\left(h^{2}+\tau^{2}\right)^{2} \\
& +C \tau\left(\left\|e^{n+1}\right\|_{2}^{2}+\left\|e^{n}\right\|_{2}^{2}+\left\|e_{x}^{n+1}\right\|_{2}^{2}+\left\|e_{x}^{n}\right\|_{2}^{2}+\left\|\eta^{n+1}\right\|_{2}^{2}+\left\|\eta^{n}\right\|_{2}^{2}\right) .
\end{aligned}
$$

Theorem 3. Assume that the function $F(s)$ is analytic in $R^{+}, E^{0}(x) \in H_{0}^{1}$, $N^{0}(x) \in L_{2}, N^{1}(x) \in L_{2} ; f(s) \in C^{\infty}\left(R^{+}\right),|F(s)| \leq C_{1} s^{\alpha}+C_{2}, 0 \leq \alpha<\frac{3}{2}$, for $s \geq 0$, and $r=\frac{\tau}{h}<\sqrt{\frac{1}{1-2 \theta}}, 0 \leq \theta \leq \frac{1}{2}$. Suppose that the solution of problem (1.5)-(1.11) satisfies $E(x, t) \in C^{5}, N(x, t) \in C^{5}$. Then the solution of the difference problem (2.1)-(2.6) converges to the solution of problem (1.5)(1.11) with order $O\left(h^{2}+\tau^{2}\right)$ in the $L_{\infty}$ norm for $E$, and in the $L_{2}$ norm for $N$.

Proof. First, we derive an estimate of $e_{j}^{n}$. Computing the inner product of (3.3) with $\left(e_{j}^{n+1}+e_{j}^{n}\right)$ and taking the imaginary part, we have

$$
\frac{1}{\tau}\left(\left\|e^{n+1}\right\|_{2}^{2}-\left\|e^{n}\right\|_{2}^{2}\right)=\operatorname{Im}\left(R^{E}, e_{j}^{n+1}+e_{j}^{n}\right)+P_{3},
$$


where

$$
\begin{aligned}
P_{3}=\operatorname{Im}( & \frac{1}{4}(N(j, n)+N(j, n+1)) \frac{F\left(|E(j, n+1)|^{2}\right)-F\left(|E(j, n)|^{2}\right)}{|E(j, n+1)|^{2}-|E(j, n)|^{2}} \\
& \cdot(E(j, n+1)+E(j, n)) \\
& \left.\quad-\frac{1}{4}\left(N_{j}^{n}+N_{j}^{n+1}\right) \frac{F\left(\left|E_{j}^{n+1}\right|^{2}\right)-F\left(\left|E_{j}^{n}\right|^{2}\right)}{\left|E_{j}^{n+1}\right|^{2}-\left|E_{j}^{n}\right|^{2}}\left(E_{j}^{n+1}+E_{j}^{n}\right), e_{j}^{n+1}+e_{j}^{n}\right) \\
=\operatorname{Im}\left(\frac{1}{4}\left(\eta_{j}^{n}+\eta_{j}^{n+1}\right) \frac{F\left(\left|E_{j}^{n+1}\right|^{2}\right)-F\left(\left|E_{j}^{n}\right|^{2}\right)}{\left|E_{j}^{n+1}\right|^{2}-\left|E_{j}^{n}\right|^{2}}\left(E_{j}^{n+1}+E_{j}^{n}\right), e_{j}^{n+1}+e_{j}^{n}\right) & \\
+\operatorname{Im}\left(\frac{1}{4}(N(j, n)+N(j, n+1))\right. & \cdot\left(\frac{F\left(|E(j, n+1)|^{2}\right)-F\left(|E(j, n)|^{2}\right)}{|E(j, n+1)|^{2}-|E(j, n)|^{2}}-\frac{F\left(\left|E_{j}^{n+1}\right|^{2}\right)-F\left(\left|E_{j}^{n}\right|^{2}\right)}{\left|E_{j}^{n+1}\right|^{2}-\left|E_{j}^{n}\right|^{2}}\right) \\
& \left.\cdot\left(E_{j}^{n+1}+E_{j}^{n}\right), e_{j}^{n+1}+e_{j}^{n}\right) .
\end{aligned}
$$

Using the inequalities (4.2), (4.3) in the Supplement, and Lemma 3, we obtain

$$
\left.\left|P_{3}\right| \leq C\left\|\eta^{n+1}\right\|_{2}^{2}+\left\|\eta^{n}\right\|_{2}^{2}+\left\|e^{n+1}\right\|_{2}^{2}+\left\|e^{n}\right\|_{2}^{2}\right) .
$$

It is easy to obtain the estimate

$$
\begin{aligned}
\left|\operatorname{Im}\left(R^{E}, e_{j}^{n+1}+e_{j}^{n}\right)\right| & =\left|\operatorname{Im}\left(O\left(h^{2}+\tau^{2}\right), e_{j}^{n+1}+e_{j}^{n}\right)\right| \\
& \leq C\left(\left(h^{2}+\tau^{2}\right)^{2}+\left\|e^{n+1}\right\|_{2}^{2}+\left\|e^{n}\right\|_{2}^{2}\right) .
\end{aligned}
$$

Thus, it follows from (3.14), (3.15), and (3.16) that

$$
\begin{aligned}
\left\|e^{n+1}\right\|_{2}^{2}-\left\|e^{n}\right\|_{2}^{2} \leq & C \tau\left(\left\|\eta^{n+1}\right\|_{2}^{2}+\left\|\eta^{n}\right\|_{2}^{2}+\left\|e^{n+1}\right\|_{2}^{2}+\left\|e^{n}\right\|_{2}^{2}\right) \\
& +C \tau\left(h^{2}+\tau^{2}\right)^{2}
\end{aligned}
$$

Computing the inner product of (3.4) with $U_{j}^{n+\frac{1}{2}}+U_{j}^{n-\frac{1}{2}}$ and using (3.2), we have

$$
\begin{aligned}
\left(\left(U_{j}^{n+\frac{1}{2}}\right)_{x \bar{x} \bar{t}}, U_{j}^{n+\frac{1}{2}}+U_{j}^{n-\frac{1}{2}}\right)-(1-2 \theta)\left(\eta_{j}^{n},\left(U_{j}^{n+\frac{1}{2}}+U_{j}^{n-\frac{1}{2}}\right)_{x \bar{x}}\right) \\
\quad-\theta\left(\eta_{j}^{n+1}+\eta_{j}^{n-1},\left(U_{j}^{n+\frac{1}{2}}+U_{j}^{n-\frac{1}{2}}\right)_{x \bar{x}}\right) \\
\quad=\left(R^{N}, U_{j}^{n+\frac{1}{2}}+U_{j}^{n-\frac{1}{2}}\right)+\left(F\left(|E(j, n)|^{2}\right)-F\left(\left|E_{j}^{n}\right|^{2}\right),\left(U_{j}^{n+\frac{1}{2}}+U_{j}^{n-\frac{1}{2}}\right)_{x \bar{x}}\right)
\end{aligned}
$$

where

$$
\begin{aligned}
-\left(\left(U_{j}^{n+\frac{1}{2}}\right)_{x \bar{x} \bar{t}}, U_{j}^{n+\frac{1}{2}}+U_{j}^{n-\frac{1}{2}}\right) & =\frac{1}{\tau}\left(\left(U_{j}^{n+\frac{1}{2}}-U_{j}^{n-\frac{1}{2}}\right)_{x},\left(U_{j}^{n+\frac{1}{2}}+U_{j}^{n-\frac{1}{2}}\right)_{x}\right) \\
& =\frac{1}{\tau}\left(\left\|U_{j}^{n+\frac{1}{2}}\right\|_{2}^{2}-\left\|\left(U_{j}^{n-\frac{1}{2}}\right)_{x}\right\|_{2}^{2}\right)
\end{aligned}
$$




$$
\begin{aligned}
& \tau\left(\eta_{j}^{n},\left(U_{j}^{n+\frac{1}{2}}+U_{j}^{n-\frac{1}{2}}\right)_{x \bar{x}}\right)=\tau\left(\eta_{j}^{n},\left(\eta_{j}^{n+1}\right)_{\bar{t}}+\left(\eta_{j}^{n}\right)_{\bar{t}}\right) \\
& =h \sum_{j=1}^{J}\left(\eta_{j}^{n+1} \eta_{j}^{n}-\eta_{j}^{n} \eta_{j}^{n-1}\right) \text {, } \\
& \tau\left(\eta_{j}^{n+1}+\eta_{j}^{n-1},\left(U_{j}^{n+\frac{1}{2}}+U_{j}^{n-\frac{1}{2}}\right)_{x \bar{x}}\right)=\left\|\eta^{n+1}\right\|_{2}^{2}-\left\|\eta^{n-1}\right\|_{2}^{2}, \\
& \tau\left(F\left(|E(j, n)|^{2}\right)-F\left(\left|E_{j}^{n}\right|^{2}\right),\left(U_{j}^{n+\frac{1}{2}}+U_{j}^{n-\frac{1}{2}}\right)_{x \bar{x}}\right) \\
& =h \sum_{j=1}^{J}\left[F\left(|E(j, n)|^{2}\right)-F\left(\left|E_{j}^{n}\right|^{2}\right)\right]\left(\eta_{j}^{n+1}-\eta_{j}^{n-1}\right) \\
& =h \sum_{j=1}^{J}\left[F\left(|E(j, n+1)|^{2}\right)-F\left(\left|E_{j}^{n+1}\right|^{2}\right)\right]\left(\eta_{j}^{n+1}+\eta_{j}^{n}\right) \\
& -h \sum_{j=1}^{J}\left[F\left(|E(j, n)|^{2}\right)-F\left(\left|E_{j}^{n}\right|^{2}\right)\right]\left(\eta_{j}^{n}+\eta_{j}^{n-1}\right) \\
& -h \sum_{j=1}^{J}\left[F\left(|E(j, n+1)|^{2}\right)-F\left(|E(j, n)|^{2}\right)\right. \\
& \left.-F\left(\left|E_{j}^{n+1}\right|^{2}\right)+F\left(\left|E_{j}^{n}\right|^{2}\right)\right]\left(\eta_{j}^{n+1}+\eta_{j}^{n}\right), \\
& \left|\tau\left(R^{N}, U_{j}^{n+\frac{1}{2}}+U_{j}^{n-\frac{1}{2}}\right)\right| \leq C \tau\left(h^{2}+\tau^{2}\right)^{2}+C \tau\left(\left\|U^{n+\frac{1}{2}}\right\|_{2}^{2}+\left\|U^{n-\frac{1}{2}}\right\|_{2}^{2}\right) .
\end{aligned}
$$

Thus,

$$
\begin{aligned}
& \left\|U_{x}^{n+\frac{1}{2}}\right\|_{2}^{2}-\left\|U_{x}^{n-\frac{1}{2}}\right\|_{2}^{2}+(1-2 \theta) h \sum_{j=1}^{J} \eta_{j}^{n+1} \eta_{j}^{n}-(1-2 \theta) h \sum_{j=1}^{J} \eta_{j}^{n} \eta_{j}^{n-1} \\
& \quad+\theta\left(\left\|\eta^{n+1}\right\|_{2}^{2}-\left\|\eta^{n-1}\right\|_{2}^{2}\right)+h \sum_{j=1}^{J}\left[F\left(|E(j, n+1)|^{2}\right)-F\left(\left|E_{j}^{n+1}\right|^{2}\right)\right]\left(\eta_{j}^{n+1}+\eta_{j}^{n}\right) \\
& \quad-h \sum_{j=1}^{J}\left[F\left(|E(j, n)|^{2}\right)-F\left(\left|E_{j}^{n}\right|^{2}\right)\right]\left(\eta_{j}^{n}+\eta_{j}^{n-1}\right) \\
& \leq P_{2}^{n+\frac{1}{2}}+C \tau\left(h^{2}+\tau^{2}\right)^{2}+C \tau\left(\left\|U^{n+\frac{1}{2}}\right\|_{2}^{2}+\left\|U_{x}^{n-\frac{1}{2}}\right\|_{2}^{2}\right) .
\end{aligned}
$$

We now compute the inner product of (3.3) with $\tau\left(e_{j}^{n+1}\right)_{\bar{t}}$ and take the real part. There results the equality

$$
-\frac{1}{2}\left(\left\|e_{x}^{n+1}\right\|_{2}^{2}-\left\|e_{x}^{n}\right\|_{2}^{2}\right)=\operatorname{Re}\left(R^{E}, e_{j}^{n+1}-e_{j}^{n}\right)+\frac{1}{4} P_{1}^{n+\frac{1}{2}}
$$

Using Lemma 5, we have

$$
\begin{aligned}
2\left(\left\|e_{x}^{n+1}\right\|_{2}^{2}-\left\|e_{x}^{n}\right\|_{2}^{2}\right) \leq & -P_{1}^{n+\frac{1}{2}}+C \tau\left(h^{2}+\tau^{2}\right)^{2} \\
& +C \tau\left(\left\|e_{x}^{n+1}\right\|_{2}^{2}+\left\|e_{x}^{n}\right\|_{2}^{2}+\left\|e^{n+1}\right\|_{2}^{2}+\left\|e^{n}\right\|_{2}^{2}\right. \\
& \left.+\left\|\eta^{n+1}\right\|_{2}^{2}+\left\|\eta^{n+1}\right\|_{2}^{2}\right) .
\end{aligned}
$$


Multiplying (3.17) by a positive constant $C_{\varepsilon}$ and adding the result to the sum of (3.19) and (3.20), we obtain

$$
L^{n+\frac{1}{2}} \leq L^{n-\frac{1}{2}}+C \tau\left(h^{2}+\tau^{2}\right)^{2}+C \tau G^{n+\frac{1}{2}}-\left(P_{1}^{n+\frac{1}{2}}-P_{2}^{n+\frac{1}{2}}\right),
$$

where

$$
\begin{aligned}
L^{n+\frac{1}{2}}= & C_{\varepsilon}\left\|e^{n+1}\right\|_{2}^{2}+2\left\|e_{x}^{n+1}\right\|_{2}^{2}+\left\|U_{x}^{n+1}\right\|_{2}^{2} \\
& +(1-2 \theta) h \sum_{j=1}^{J} \eta_{j}^{n+1} \eta_{j}^{n}+\theta\left(\left\|\eta^{n+1}\right\|_{2}^{2}+\left\|\eta^{n}\right\|_{2}^{2}\right) \\
& +h \sum_{j=1}^{J}\left[F\left(|E(j, n+1)|^{2}\right)-F\left(\left|E_{j}^{n+1}\right|^{2}\right)\right]\left(\eta_{j}^{n+1}+\eta_{j}^{n}\right), \\
G^{n+\frac{1}{2}}= & \left\|e_{x}^{n+1}\right\|_{2}^{2}+\left\|e_{x}^{n}\right\|_{2}^{2}-\left\|e^{n+1}\right\|_{2}^{2}+\left\|e^{n}\right\|_{2}^{2} \\
& +\left\|U_{x}^{n+\frac{1}{2}}\right\|_{2}^{2}+\left\|U_{x}^{n-\frac{1}{2}}\right\|_{2}^{2}+\left\|\eta^{n+1}\right\|_{2}^{2}+\left\|\eta^{n}\right\|_{2}^{2} .
\end{aligned}
$$

It follows from Lemma 4 that

$$
\begin{aligned}
L^{n+\frac{1}{2}} & \leq L^{n-\frac{1}{2}}+C \tau\left(h^{2}+\tau^{2}\right)^{2}+C \tau G^{n+\frac{1}{2}} \\
& \leq L^{-\frac{1}{2}}+C\left(h^{2}+\tau^{2}\right)^{2}+C \tau \sum_{l=0}^{n} G^{l+\frac{1}{2}} \\
& \leq C\left(h^{2}+\tau^{2}\right)^{2}+C \tau \sum_{l=0}^{n} G^{l+\frac{1}{2}} .
\end{aligned}
$$

Lemma 2 yields

$$
\begin{aligned}
L^{n+\frac{1}{2}} \geq & C_{\ell}\left\|e^{n+1}\right\|_{2}^{2}+2\left\|e_{x}^{n+1}\right\|_{2}^{2}+\frac{1}{C_{1}}\left\|U_{x}^{n+\frac{1}{2}}\right\|_{2}^{2} \\
& +\left(\frac{1-2 \theta}{2 C_{1}}+\theta\right)\left(\left\|\eta^{n+1}\right\|_{2}^{2}+\left\|\eta^{n}\right\|_{2}^{2}\right) \\
& +h \sum_{j=1}^{J}\left[F\left(|E(j, n+1)|^{2}\right)-F\left(\left|E_{j}^{n+1}\right|^{2}\right)\right]\left(\eta_{j}^{n+1}+\eta_{j}^{n}\right),
\end{aligned}
$$

while

(3.24)

$$
\begin{aligned}
& \left|h \sum_{j=1}^{J}\left[F\left(|E(j, n+1)|^{2}\right)-F\left(\left|E_{j}^{n+1}\right|^{2}\right)\right]\left(\eta_{j}^{n+1}+\eta_{j}^{n}\right)\right| \\
& \quad \leq \varepsilon_{3}\left(\left\|\eta^{n+1}\right\|_{2}^{2}+\left\|\eta^{n}\right\|_{2}^{2}\right)+\frac{1}{\varepsilon_{3}} h \sum_{j=1}^{J}\left|F\left(|E(j, n+1)|^{2}\right)-F\left(\left|E_{j}^{n+1}\right|^{2}\right)\right|^{2} \\
& \quad \leq \varepsilon_{3}\left(\left\|\eta^{n+1}\right\|_{2}^{2}+\left\|\eta^{n}\right\|_{2}^{2}\right)+\frac{1}{\varepsilon_{3}} h \sum_{j=1}^{J}\left|f\left(\xi_{3}\right)\right|^{2}\left(|E(j, n+1)|+\left|E_{j}^{n+1}\right|\right)^{2}\left|e_{j}^{n+1}\right|^{2} \\
& \quad \leq \varepsilon_{3}\left(\left\|\eta^{n+1}\right\|_{2}^{2}+\left\|\eta^{n}\right\|_{2}^{2}\right)+\frac{C_{3}}{\varepsilon_{3}}\left\|e^{n+1}\right\|_{2}^{2} .
\end{aligned}
$$


Substituting (3.24) into (3.23) and choosing $\varepsilon_{3}=\frac{1}{2}\left(\frac{1-2 \theta}{2 C_{1}}+\theta\right)$ and $C_{\varepsilon}=\frac{2 C_{3}}{\varepsilon_{3}}$, we have

$$
\begin{aligned}
L^{n+\frac{1}{2}} \geq & \frac{2 C_{3}}{\frac{1-2 \theta}{2 C_{1}}+\theta}\left\|e^{n+1}\right\|_{2}^{2}+2\left\|e_{x}^{n+1}\right\|_{2}^{2}+\frac{1}{C_{1}}\left\|U_{x}^{n+\frac{1}{2}}\right\|_{2}^{2} \\
& +\frac{1}{2}\left(\frac{1-2 \theta}{2 C_{1}}+\theta\right)\left(\left\|\eta^{n+1}\right\|_{2}^{2}+\left\|\eta^{n}\right\|_{2}^{2}\right) \\
\geq & C\left(\left\|e^{n+1}\right\|_{2}^{2}+\left\|e_{x}^{n+1}\right\|_{2}^{2}+\left\|U_{x}^{n+\frac{1}{2}}\right\|_{2}^{2}+\left\|\eta^{n+1}\right\|_{2}^{2}+\left\|\eta^{n}\right\|_{2}^{2}\right) .
\end{aligned}
$$

Thus, combining (3.25) and (3.22) yields

$$
\begin{gathered}
\left\|e^{n+1}\right\|_{2}^{2}+\left\|e_{x}^{n+1}\right\|_{2}^{2}+\left\|\eta^{n+1}\right\|_{2}^{2}+\left\|\eta^{n}\right\|_{2}^{2}+\left\|U_{x}^{n+\frac{1}{2}}\right\|_{2}^{2} \\
\leq C\left(h^{2}+\tau^{2}\right)^{2}+C \tau \sum_{l=0}^{n} G^{l+\frac{1}{2}}
\end{gathered}
$$

which is equivalent to

$$
G^{n+\frac{1}{2}} \leq 2 C\left(h^{2}+\tau^{2}\right)^{2}+2 C \tau \sum_{l=0}^{n} G^{l+\frac{1}{2}},
$$

i.e.,

$$
G^{n+\frac{1}{2}} \leq C\left(h^{2}+\tau^{2}\right)^{2}+C \tau \sum_{l=0}^{n-1} G^{l+\frac{1}{2}} .
$$

Using the discrete Gronwall inequality [12], we obtain

$$
G^{n+\frac{1}{2}} \leq C\left(h^{2}+\tau^{2}\right)^{2}, \quad 0 \leq n \leq \frac{T}{\tau}
$$

where $C_{T}$ is a constant depending on $T$.

It follows from the definition of $G^{n+\frac{1}{2}}$ that

$$
\left\|e^{n}\right\|_{\infty} \leq C\left(\left\|e^{n}\right\|_{2}+\left\|e_{x}^{n}\right\|_{2}\right) \leq C\left(h^{2}+\tau^{2}\right)
$$

and

$$
\left\|\eta^{n}\right\|_{2} \leq C\left(h^{2}+\tau^{2}\right)
$$

This completes the proof.

Finally, it is easy to verify that all lemmas and theorems in this paper hold for the periodic initial-value problem for the generalized Zakharov equations.

\section{BIBLIOGRAPHY}

1. H. Added and S. Added, Equations of Langmuir turbulence and nonlinear Schrödinger equation: Smoothness and approximation, J. Funct. Anal. 29 (1988), 183-210.

2. I. Blalynickl-Birdla and J. Mycialski, Gaussons: Solitons of the logarithmic Schrödinger equation, Phys. Scripta 20 (1979), 539-544.

3. R. T. Bullough, P. M. Jack, P. W. Kitchenside, and R. Saunders, Solitons in laser physics, Phys. Scripta 20 (1979), 364-381.

4. Q. Chang, Conservative difference scheme for generalized nonlinear Schrödinger equations, Scientia Sinica (Series A) 26 (1983), 687-701.

5. Q. Chang and H. Jiang, A conservative difference scheme for the Zakharov equations, J. Comput. Phys. (to appear). 
6. Q. Chang and L. Xu, A numerical method for a system of generalized nonlinear Schrödinger equations, J. Comput. Math. 4 (1986), 191-199.

7. Q. Chang and G. Wang, Multigrid and adaptive algorithm for solving the nonlinear Schrödinger equations, J. Comput. Phys. 88 (1990), 362-380.

8. A. Friedman, Partial differential equations, Holt, New York, 1969.

9. R. Glassey, Convergence of an energy-preserving scheme for the Zakharov equations in one space dimension, Math. Comp. 58 (1992), 83-102.

10. __ Approximate solutions to the Zakharov equations via finite differences, J. Comput. Phys. 100 (1992), 377-383.

11. K. Konno and H. Suzuki, Self-focussing of laser beam in nonlinear media, Phys. Scripta 20 (1979), 382-386.

12. Milton Lees, Approximate solution of parabolic equations, J. Soc. Indust. Appl. Math. 7 (1959), 167-183.

13. J. C. Lopez-Marcos and J. M. Sanz-Serna, Stability and convergence in numerical analysis III: Linear investigation of nonlinear stability, IMA J. Numer. Anal. 8 (1988), 71-84.

14. A. Menikoff, The existence of unbounded solutions of the KdV equation, Comm. Pure Appl. Math. 25 (1972), 407-432.

15. G. L. Payne, D. R. Nicholson, and R. M. Downie, Numerical solution of the Zakharov equations, J. Comput. Phys. 50 (1983), 482-498.

16. T. Ortega and J. M. Sanz-Serna, Nonlinear stability and convergence of finite-difference methods for the "good" Boussinesq equation, Numer. Math. 58 (1990), 215-229.

17. S. Schochet and M. Weinstein, The nonlinear Schrödinger limit of the Zakharov equations governing Langmuir turbulence, Comm. Math. Phys. 106 (1986), 569-580.

18. W. A. Strauss, Mathematical aspects of classical nonlinear field equations, Lecture Notes in Phys., vol. 98, Springer, Berlin, 1979, pp. 123-149.

19. C. Sulem and P. L. Sulem, Regularity properties for the equations of Langmuir turbulence, C. R. Acad. Sci. Paris Sér. A Math. 289 (1979), 173-176.

20. V. E. Zakharov, Collapse of Langmuir waves, Soviet Phys. JETP 35 (1972), 908-912.

21. P. K. C. Wang, A class of multidimensional nonlinear Langmuir waves, J. Math. Phys. 19 (1978), 1286.

Institute of Applied Mathematics, The Chinese Academy of Sciences, Beijing, 100080, China; and Laboratory of Computational Physics, IAPCM, P.O. Box 8009, Beining 100088, CHINA

E-mail address: qschang@bepc2.ihep.ac.cn

Institute of Applied Physics and Computational Mathematics, P.O. Box 8009, Beijing 100088, CHINA

Department of Computer Science, University of Waterloo, Waterloo, Ontario, CANADA N2L 3G1

E-mail address: hjiang@yoho.uwaterloo.ca 


\section{Supplement to}

\section{FINITE DIFFERENCE METHOD FOR GENERALIZED ZAKHAROV EQUATIONS}

\section{QIANSHUN CHANG, BOLING GUO, AND HONG JIANG}

In this section, long and highly technical proofs of two Lemmas in Section 3 are given.

Proof of Lemma 4. Direct computation implies that

$$
\begin{aligned}
& P_{1}^{n+\frac{1}{2}}-P_{2}^{n+\frac{1}{2}} \\
& =\operatorname{Re}\left\{h \sum _ { j = 1 } ^ { J } \left[(N(j, n)+N(j, n+1))\left(F\left(|E(j, n+1)|^{2}\right)+F\left(|E(j, n)|^{2}\right)\right)\right.\right. \\
& -(N(j, n)+N(j, n+1)) \frac{F\left(|E(j, n+1)|^{2}\right)-F\left(|E(j, n)|^{2}\right)}{|E(j, n+1)|^{2}-|E(j, n)|^{2}}(E(j, n+1)+E(j, n)) \\
& \cdot\left(\overline{E_{j}^{n+1}-E_{j}^{n}}\right)-\left(N_{j}^{n}+N_{j}^{n+1}\right) \frac{F\left(\left|E_{j}^{n+1}\right|^{2}\right)-F\left(\left|E_{j}^{n}\right|^{2}\right)}{\left|E_{j}^{n+1}\right|^{2}-\left|E_{j}^{n}\right|^{2}}\left(E_{j}^{n+1}+E_{j}^{n}\right) \\
& \left.\left.\cdot(\overline{E(j, n+1)-E(j, n)})+\left(N_{j}^{n}+N_{j}^{n+1}\right)\left(F\left(\left|E_{j}^{n+1}\right|^{2}\right)-F\left(\left|E_{j}^{n}\right|^{2}\right)\right)\right]\right\} \\
& \left.-h \sum_{j=1}^{J}\left[F\left(|E(j, n+1)|^{2}\right)-F\left(|E(j, n)|^{2}\right)-F\left(\left|E_{j}^{n+1}\right|^{2}\right)+\left.F\left(\mid E_{j}^{n}\right)\right|^{2}\right)\right] \\
& \cdot\left[N(j, n+1)+N(j, n)-N_{j}^{n+1}-N_{j}^{n}\right] \\
& =\operatorname{Re}\left\{h \sum _ { j = 1 } ^ { J } \left[\left(N_{j}^{n+1}+N_{j}^{n}\right)(\overline{E(j, n+1)-E(j, n)})-(N(j, n)+N(j, n+1))\right.\right. \\
& \cdot\left(\overline{\left.E_{j}^{n+1}-E_{j}^{n}\right)}\right]\left[\frac{F\left(|E(j, n+1)|^{2}-F\left(|E(j, n)|^{2}\right)\right.}{|E(j, n+1)|^{2}-|E(j, n)|^{2}}(E(j, n+1)+E(j, n))\right. \\
& \left.\left.-\frac{F\left(\left|E_{j}^{n+1}\right|^{2}\right)-F\left(\left|E_{j}^{n}\right|^{2}\right)}{\left|E_{j}^{n+1}\right|^{2}-\left|E_{j}^{n}\right|^{2}}\left(E_{j}^{n+1}+E_{j}^{n}\right)\right]\right\} \\
& =\operatorname{Re}\left\{h \sum _ { j = 1 } ^ { J } \left[(N(j, n)+N(j, n+1))\left(\overline{e_{j}^{n+1}-e_{j}^{n}}\right)-(\overline{E(j, n+1)-E(j, n)}\right.\right. \\
& \left.\cdot\left(\eta_{j}^{n+1}+\eta_{j}^{n}\right)\right]\left[\frac{F\left(\left|E_{j}^{n+1}\right|^{2}\right)-F\left(\left|E_{j}^{n}\right|^{2}\right)}{\left|E_{j}^{n+1}\right|^{2}-\left|E_{j}^{n}\right|^{2}}\left(e_{j}^{n+1}+e_{j}^{n}\right)\right.
\end{aligned}
$$



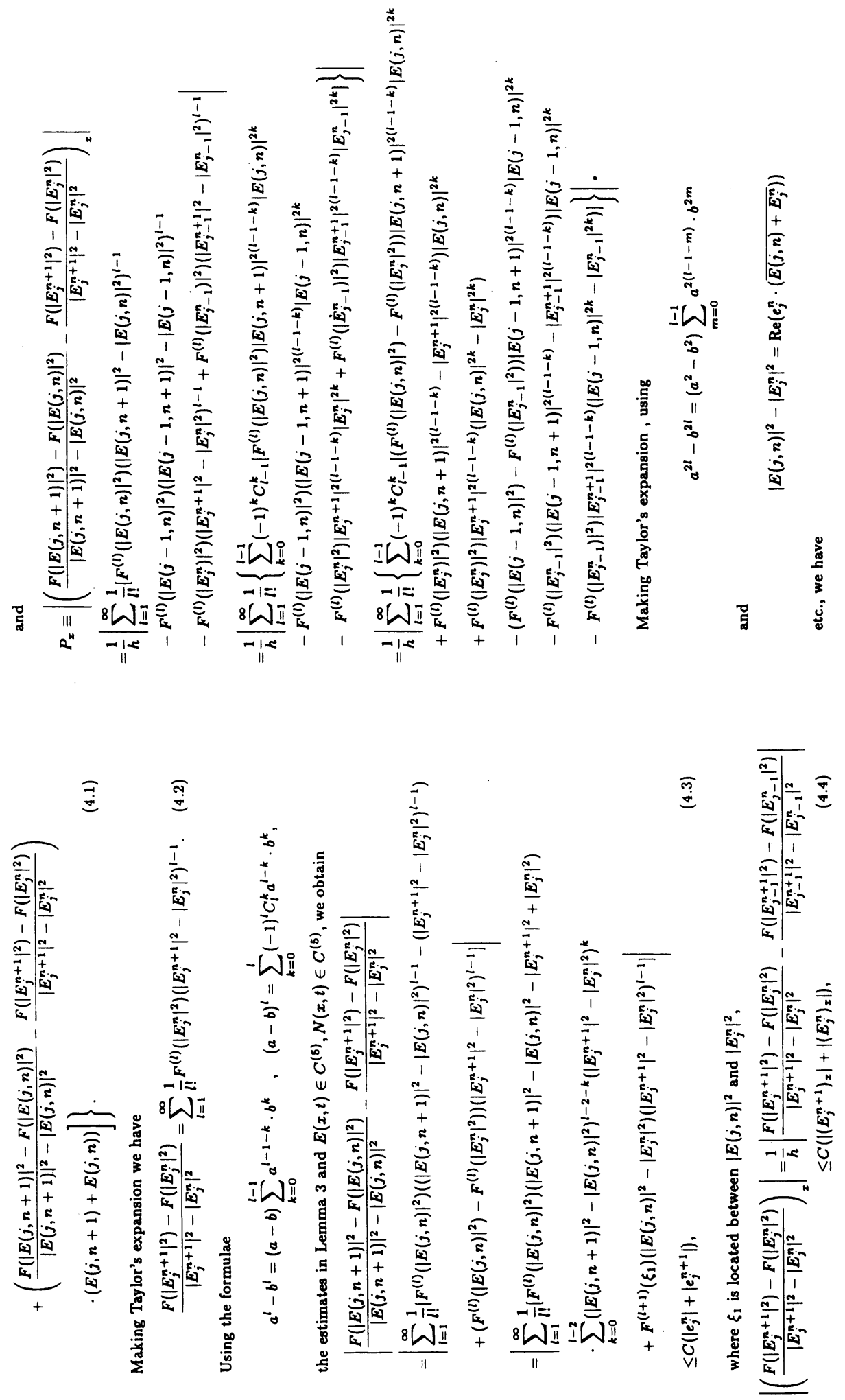

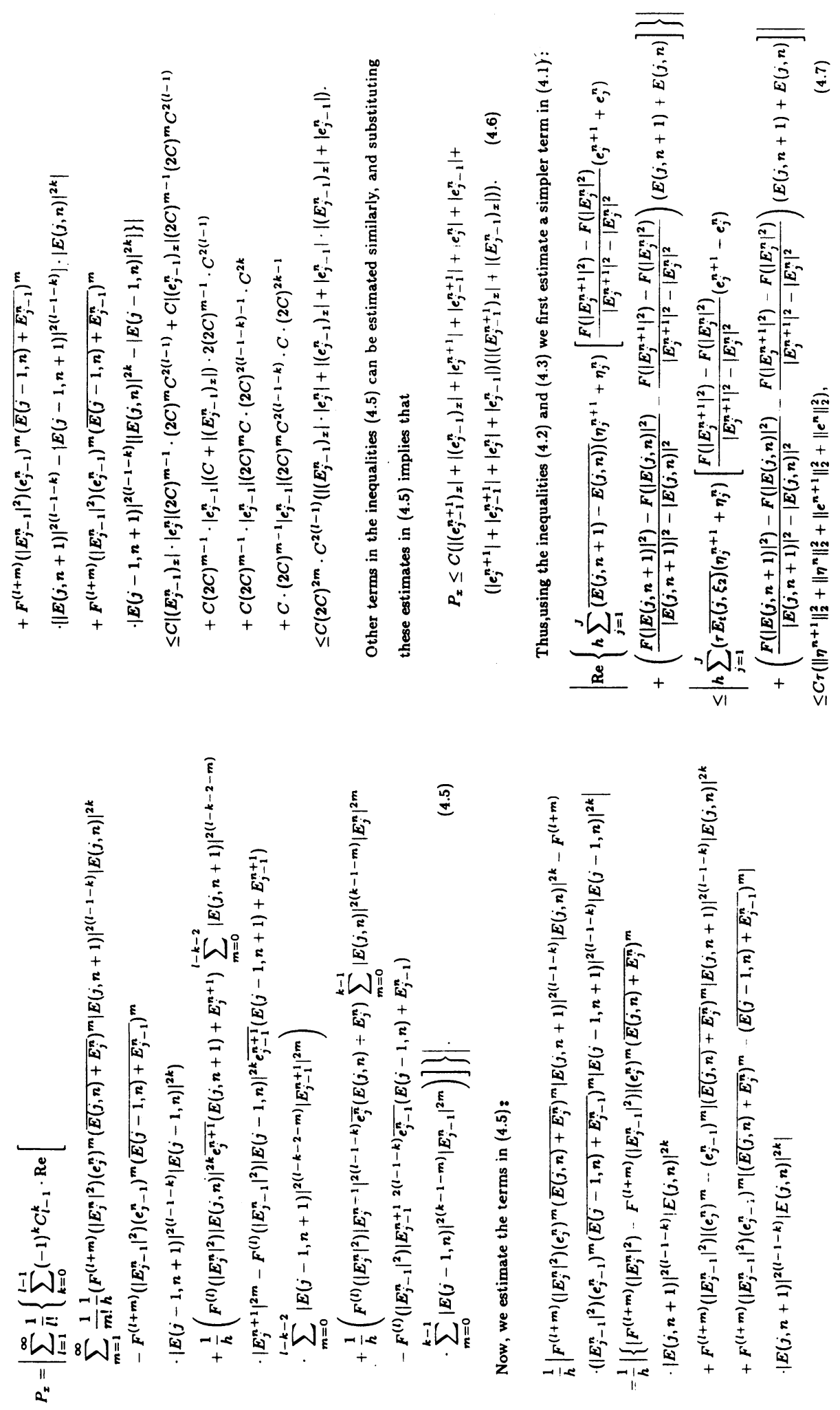


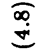
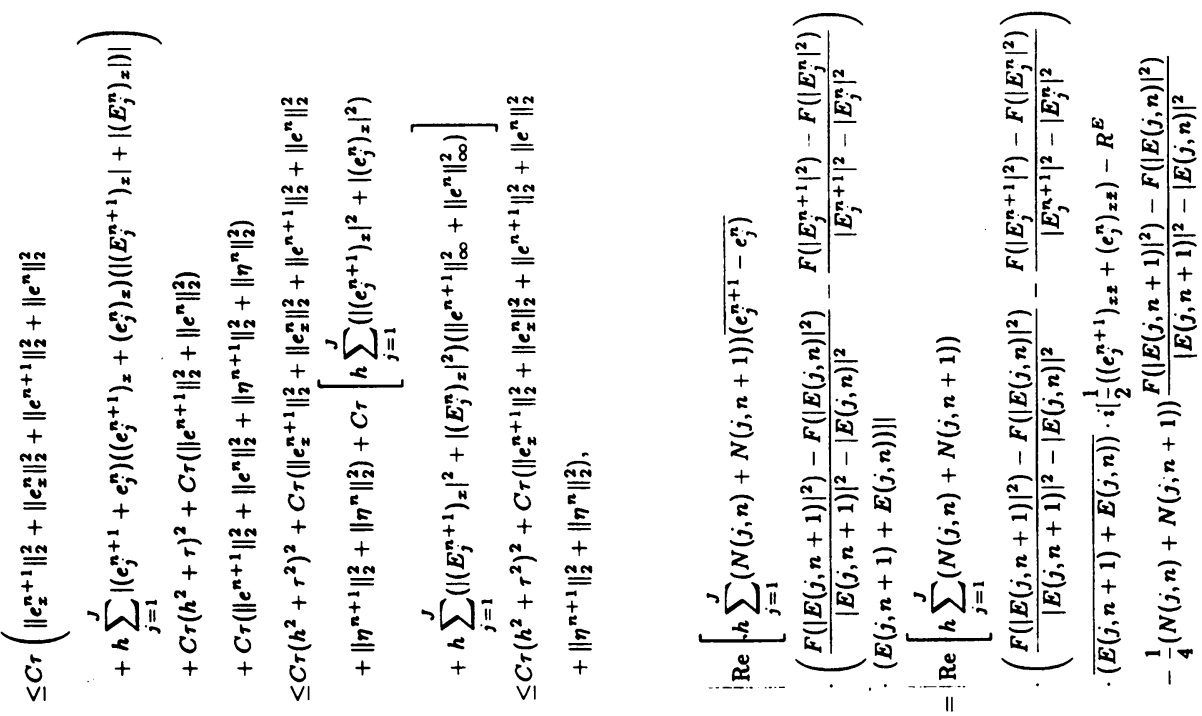

ซ్

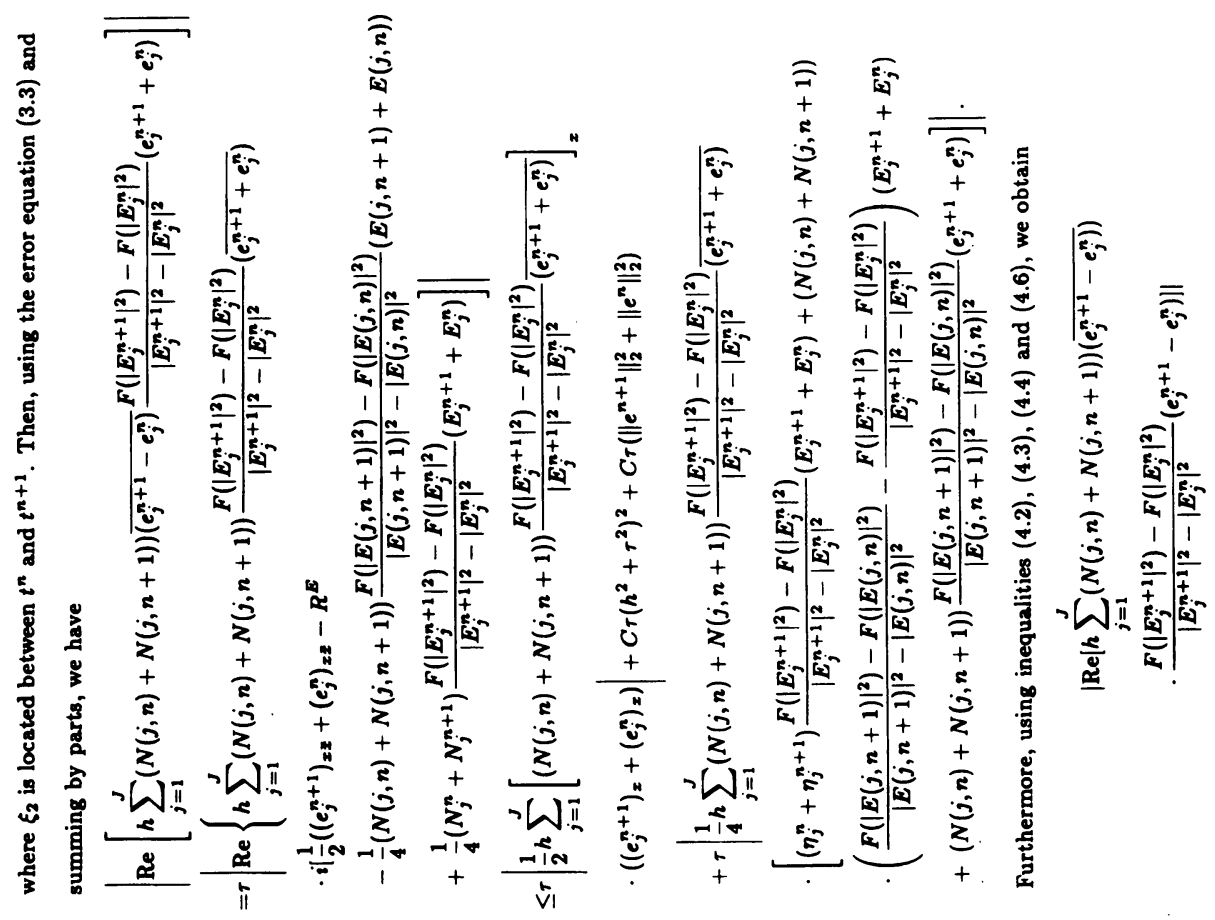




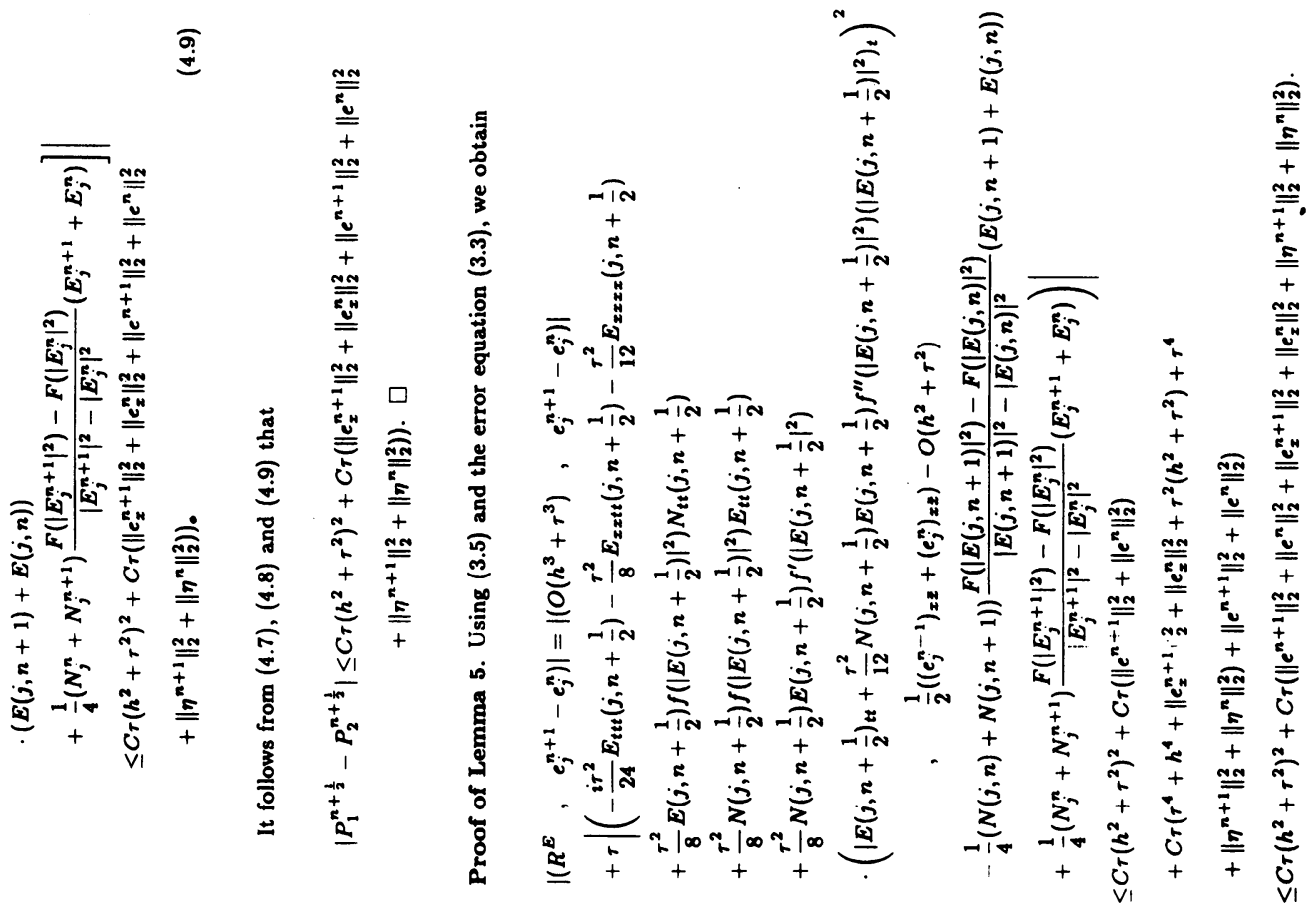

